\title{
Out-of-hospital cardiac arrest in high-rise buildings: delays to patient care and effect on survival
}

\author{
Ian R. Drennan ACP, Ryan P. Strum PCP BSc, Adam Byers BSc, Jason E. Buick PCP MSc, Steve Lin MDCM MSc, \\ Sheldon Cheskes MD, Samantha Hu, Laurie J. Morrison MD MSc; for the Rescu Investigators
}

CMAJ Podcasts: author interview at https://soundcloud.com/cmajpodcasts/150544-res

See also page 399 and www.cmaj.ca/lookup/doi/10.1503/cmaj.151044

\begin{abstract}
Background: The increasing number of people living in high-rise buildings presents unique challenges to care and may cause delays for 911-initiated first responders (including paramedics and fire department personnel) responding to calls for out-ofhospital cardiac arrest. We examined the relation between floor of patient contact and survival after cardiac arrest in residential buildings.
\end{abstract}

Methods: We conducted a retrospective observational study using data from the Toronto Regional RescuNet Epistry database for the period January 2007 to December 2012. We included all adult patients ( $\geq 18 \mathrm{yr}$ ) with out-of-hospital cardiac arrest of no obvious cause who were treated in private residences. We excluded cardiac arrests witnessed by 911-initiated first responders and those with an obvious cause. We used multivariable logistic regression to determine the effect on survival of the floor of patient con-

$\mathrm{M}$ ore than 400000 out-of-hospital cardiac arrests occur annually in North America. ${ }^{1,2}$ Despite considerable effort to improve resuscitation care, survival to hospital discharge in most communities remains below $10 \% .^{2}$ Rapid defibrillation and high-quality cardiopulmonary resuscitation (CPR) are essential for survival, with an absolute decrease in survival of $7 \%$ to $10 \%$ for each 1-minute delay to defibrillation. ${ }^{3-5}$

Recently, there has been a dramatic increase in the number of people living in high-rise buildings (e.g., a 13\% relative increase in Toronto from 2006 to $2011^{6,7}$ ). As more high-rise buildings are constructed in urban centres across Canada, the number of 911 calls for emergency medical services in high-rise buildings will also continue to increase. Furthermore, over $40 \%$ of homeowners tact, with adjustment for standard Utstein variables.

Results: During the study period, 7842 cases of out-of-hospital cardiac arrest met the inclusion criteria, of which 5998 (76.5\%) occurred below the third floor and 1844 (23.5\%) occurred on the third floor or higher. Survival was greater on the lower floors $(4.2 \%$ v. $2.6 \%, p=0.002)$. Lower adjusted survival to hospital discharge was independently associated with higher floor of patient contact, older age, male sex and longer 911 response time. In an analysis by floor, survival was $0.9 \%$ above floor 16 (i.e., below the $1 \%$ threshold for futility), and there were no survivors above the 25 th floor.

Interpretation: In high-rise buildings, the survival rate after out-of-hospital cardiac arrest was lower for patients residing on higher floors. Interventions aimed at shortening response times to treatment of cardiac arrest in high-rise buildings may increase survival.

over the age of 65 years reside in high-rise buildings. ${ }^{8}$ These older residents have higher risks for a number of serious medical conditions, including cardiac arrest. Cardiac arrests that occur in highrise buildings pose unique challenges for 911initiated first responders. Building access issues, elevator delays and extended distance from the location of the responding vehicle on scene to the patient can all contribute to longer times to patient contact and, ultimately, longer times to initiation of resuscitation. Previous research has shown that longer 911 response times result in decreased patient survival after cardiac arrest, ${ }^{9,10}$ but response times are traditionally measured from the time a call is received by the 911 dispatch centre to when the response vehicle arrives on scene. This measure fails to take into account the time required for 911-initiated first responders to
Competing interests:

Steve Lin has received grant funding from the Zoll

Foundation. Sheldon

Cheskes has accepted speaker's honoraria from the Zoll Foundation and Physio Control. Sheldon Cheskes and Laurie Morrison receive salary support from the Canadian Institutes of Health

Research, the National Institutes of Health

(National Heart, Lung and Blood Institute) and the

Heart and Stroke Foundation of Canada, as lead investigators for the University of Toronto

Regional Coordinating Centre for the Resuscitation Outcomes Consortium. No other competing interests were declared.

This article has been peer reviewed.

Accepted: Nov. 13, 2015

Online: Jan. 18, 2016

Correspondence to:

Ian Drennan,

drennani@smh.ca

CMAJ 2016. DOI:10.1503/ cmaj.150544 
make patient contact once they arrive on scene. This interval can contribute substantial delays to patient treatment, in some cases more than 4 minutes, and can account for up to $28 \%$ of the total time from the 911 call to arrival of the first responders at the patient's side. ${ }^{11-14}$

There is a lack of literature describing the delay to patient contact during out-of-hospital cardiac arrests in high-rise buildings, where timesensitive, life-saving interventions matter most. Furthermore, the effect on survival of vertical delay to patient contact is unknown. As the number of high-rise buildings continues to increase and as population density rises in major urban centres, is important to determine the effect of delays to patient care in high-rise buildings on survival after cardiac arrest and to examine potential barriers to patient care in this setting.

The primary objective of this study was to compare the rate of survival to hospital discharge after out-of-hospital cardiac arrest at different vertical heights in residential buildings, specifically higher floors ( $\geq 3$ floors) relative to lower floors ( $<3$ floors), with adjustment for standard Utstein variables. ${ }^{15}$

The secondary objectives were to determine the delay to patient contact by 911-initiated first responders for cardiac arrests occurring on higher floors and to examine the use of automated external defibrillators by bystanders in private residences.

\section{Methods}

\section{Design and setting}

For this retrospective, observational study, we used data from the Toronto Regional RescuNet Epistry database, which is based upon data definitions set out by the Resuscitation Outcomes Consortium Epistry - Cardiac Arrest ${ }^{16}$ and the Strategies for Post Arrest Resuscitation Care Network ${ }^{17}$ databases. Briefly, the Toronto Regional RescuNet Epistry database is a prospective, population-based registry of consecutive out-of-hospital cardiac arrests attended by 911-initiated first responders in urban and rural regions of southern Ontario, which together have a population of more than 6.6 million residents. Data are collected from a network of 7 land-based emergency medical services agencies, local fire departments, the provincial air ambulance service and 44 participating destination hospitals. Trained data guardians enter epidemiologic data from standardized prehospital call reports and inhospital records into secured databases. The research ethics board at the lead institution (St. Michael's Hospital, Toronto) has provided ethics approval for all retrospective studies related to the Toronto Regional RescuNet Epistry database.

\section{Study population}

We included all consecutively treated adults ( $\geq 18 \mathrm{yr}$ ) with out-of-hospital cardiac arrest of no obvious cause (such as drug overdose, drowning or trauma) and therefore presumed to be of cardiac origin that occurred between Jan. 1, 2007, and Dec. 31, 2012, within the City of Toronto and the Regional Municipality of Peel. These regions were selected because of their high population density $\left(>1000 / \mathrm{km}^{2}\right) .6,18$ We excluded cardiac arrests witnessed by 911-initiated first responders, those of a traumatic nature or with another obvious cause, and those that occurred in a health care setting or public location. We obtained location classifications from prehospital ambulance call reports. Private locations were defined as apartments, condominiums, houses or townhouses. All other locations were considered public. Locations classified as "other" on the ambulance call report were considered unknown and were excluded from the analysis. The third floor was selected as the cutoff for vertical height, as this data point is currently captured on government-approved ambulance call reports. In this study, 911-initiated first responders consisted of both paramedics and fire department personnel, and 911 response time was measured from the time a call was received by the 911 dispatcher until arrival on scene of a response vehicle (either ambulance or fire department vehicle).

\section{Statistical analysis}

We calculated baseline descriptive statistics for all variables of interest. Continuous variables were summarized as median and interquartile range or mean and standard deviation, as appropriate, and categorical variables were expressed as counts and percentages. We conducted bivariable analyses to determine differences for each variable of interest in terms of floor of patient contact, as well as differences between survivors and nonsurvivors. We used the $t$ test or Wilcoxon rank-sum test, as appropriate, for continuous variables, and the $\chi^{2}$ test for categorical variables. We calculated the difference in survival by floor of contact using a $\chi^{2}$ test. We performed a subgroup analysis based on whether the cardiac arrest occurred in an apartment or in a house or townhouse. We also performed an analysis by floor of the pick-up address (rather than categorized as below versus at or above the third floor). For all analyses, a $p$ value less than 0.05 was considered statistically significant. We used multivariable logistic regression to determine independent associations between the vertical height of patient contact ( $<$ floor $3 \mathrm{v}$. $\geq$ floor 3 ) and survival to hospital discharge, with adjustment for standard Utstein variables 
(age, sex, private or public location, shockable or nonshockable initial rhythm, 911 response time, witness status and bystander CPR). ${ }^{15} \mathrm{We}$ also conducted a post hoc analysis to examine the effect of vertical height on the outcome of return of spontaneous circulation. The results of the multivariable logistic regression models are reported as odds ratios (ORs) with $95 \%$ confidence intervals (CIs). All statistical analyses were conducted with $\mathrm{R}$ software, version 3.0.2 (R Foundation for Statistical Computing).

\section{Results}

A total of 12960 out-of-hospital cardiac arrests were treated by 911-initiated first responders during the study period, of which 8216 occurred in private residences and were included in the study. Of these, $5998(73.0 \%)$ occurred below the third floor, 1844 (22.4\%) occurred at or above the third floor, and $374(4.6 \%)$ were excluded because the floor number was missing (Figure 1). Overall, there was a $20 \%$ relative increase in the annual rate of out-of-hospital cardiac arrests in private residences across the study period.

Significant differences in event characteristics were noted between cardiac arrests that occurred on higher floors and those that occurred on lower floors (Table 1). In general, cardiac arrests that occurred on the third floor or above were less likely to involve a male patient and were less often witnessed by bystanders, and an initial shockable rhythm was less often present. There was also a longer delay from the time of arrival of 911-initiated first responders on scene to patient contact on the higher floors (4.9 \pm $2.6 \min$ v. $3.0 \pm 2.0 \mathrm{~min} ; p=0.01$ ).

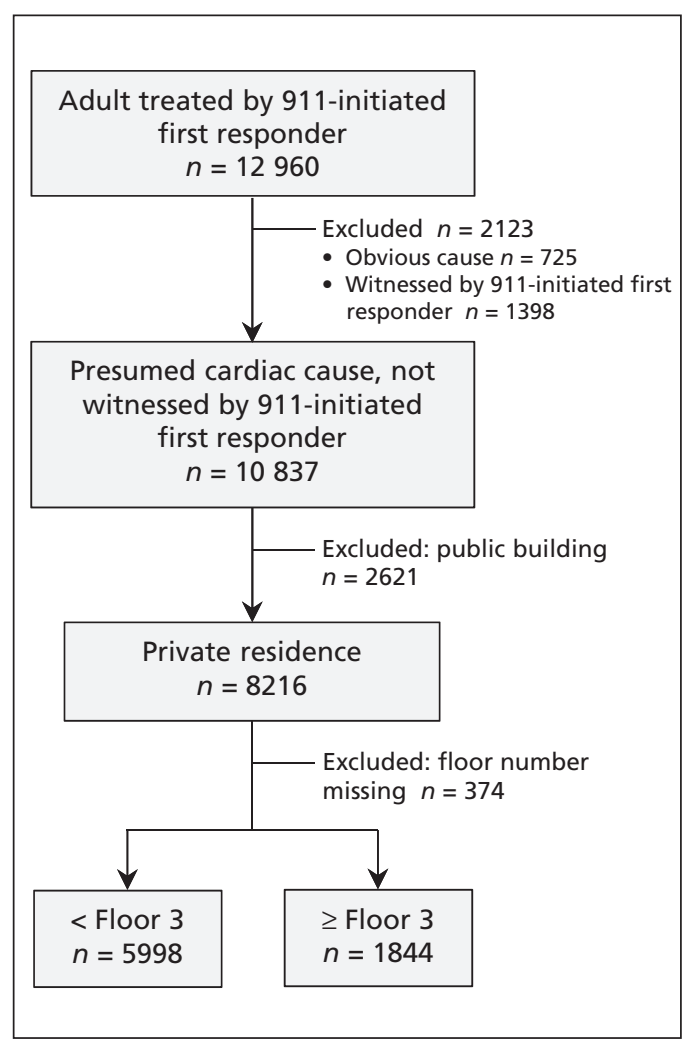

Figure 1: CONSORT diagram for flow of patients in a study of out-of-hospital cardiac arrest in private residences.

Table 1: Demographic and clinical characteristics of patients with cardiac arrest occurring in a private residence, by floor of patient contact

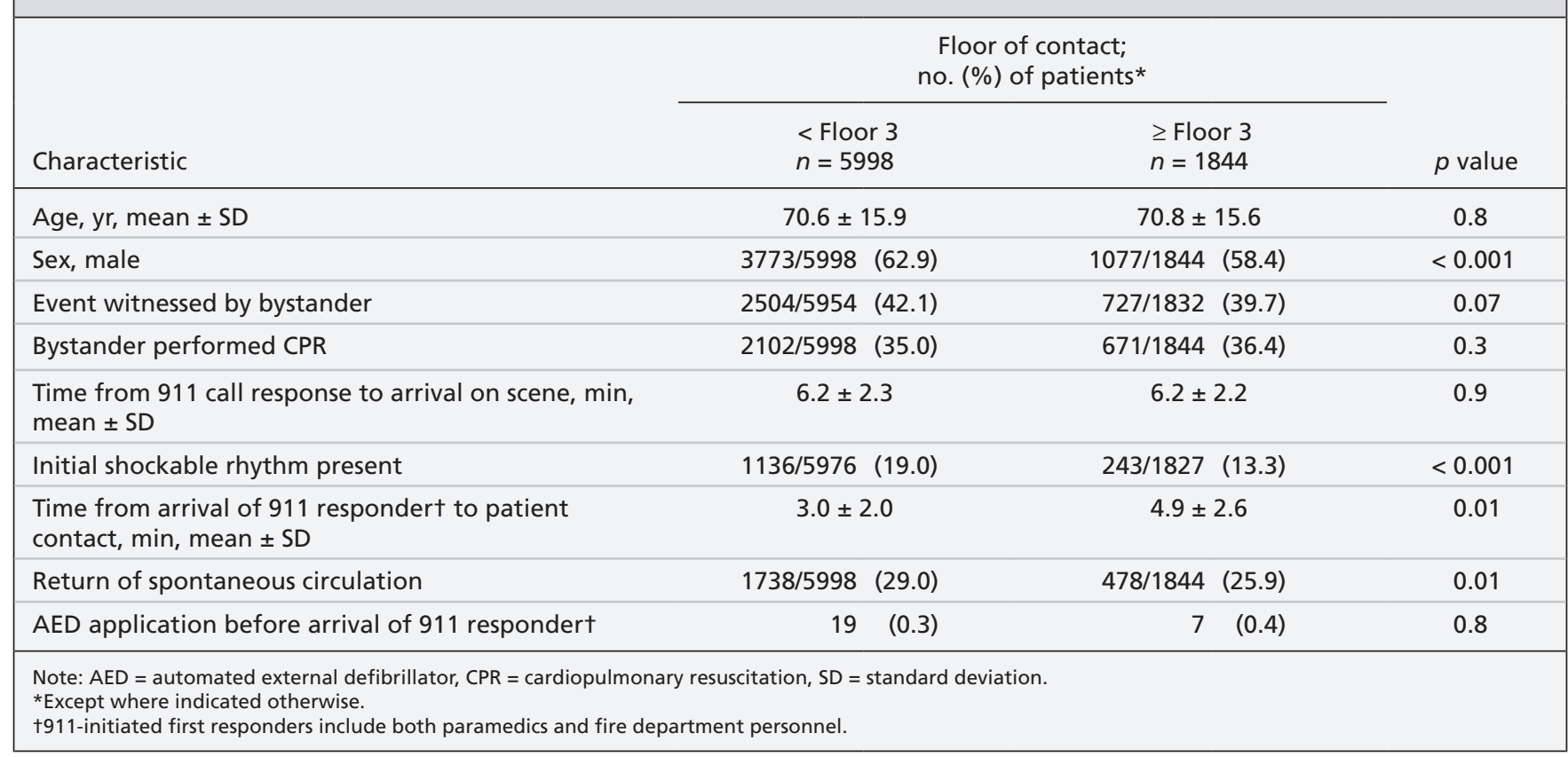


Overall, 3.8\% (300/7842) of the patients survived to hospital discharge. A total of 252 (4.2\%) of the 5998 patients with cardiac arrest below the third floor survived, compared with 48 $(2.6 \%)$ of the 1844 patients on the third floor or above $(p=0.002)$. In the subgroup analysis based on building type, there were 2363 patients living in apartment buildings and 5479 living in houses or townhouses. Of the apartment dwellers, $35(5.2 \%)$ of 667 whose cardiac arrest occurred below the third floor and $46(2.7 \%)$ of 1696 with cardiac arrest on the third floor or higher survived $(p=0.004)$. Among those dwelling in houses or townhouses, 217 (4.1\%) of 5331 whose cardiac arrest occurred below the third floor and $2(1.4 \%)$ of 148 with cardiac arrest on the third floor or higher survived $(p=0.1)$.

The unadjusted analysis showed that, compared with nonsurvivors, those who survived to hospital discharge were on average younger, their cardiac arrest had more often been witnessed by bystanders, the rate of bystander CPR was higher, and they were more likely to present in an initial shockable rhythm. Survivors also had shorter 911 response times to arrival on scene and shorter times from arrival on scene to patient contact (Table 2).

The multivariable regression analysis, with adjustment for the effects of Utstein variables, showed that cardiac arrest on higher floors ( $\geq$ floor 3) was independently associated with lower survival to hospital discharge (OR 0.70, 95\% CI 0.50-0.99). Older age (OR 0.96, 95\% CI 0.95-0.97), male sex (OR 0.72, 95\% CI 0.54 0.95 ) and longer 911 response times (OR 0.86, 95\% CI 0.79-0.92) were also associated with lower rates of survival. Patients presenting with an initial shockable rhythm (OR 10.68, 95\% CI 7.98-14.29) and bystander witness of the cardiac arrest (OR 2.93, 95\% CI 2.16-3.98) were associated with higher rates of survival to discharge, whereas performance of CPR by a bystander was not associated with survival (OR 1.07, 95\% CI 0.83-1.39) (Table 2).

In the analysis by individual floor of the pickup address, the spread of data was from floor 1 to floor 48 (median 2). The survival rate above floor 16 was $0.9 \%(2 / 216)$, and there were no survivors above floor $25(0 / 30)$. The adjusted analysis showed a statistically significant decrease in survival to hospital discharge for each floor of patient contact (OR 0.95, 95\% CI 0.91-0.99).

A total of 2216 (28.3\%) of the 7842 patients experienced return of spontaneous circulation; $478(25.9 \%)$ of 1844 at or above the third floor and $1738(29.0 \%)$ of 5998 below the third floor. After adjustment for Utstein variables, the floor of patient contact ( $\geq$ floor $3 \mathrm{v}$. $<$ floor 3) was not associated with return of spontaneous circulation (OR $0.90,95 \%$ CI 0.79-1.02) (Table 3). The results for data analyzed by every 5 floors and every 10 floors, with inclusion of the time to patient contact by 911-initiated first responders, are shown in Appendix 1 (available at www.cmaj.ca/lookup/ suppl/doi:10.1503/cmaj.150544/-/DC1).

The use of automated external defibrillators was very low, regardless of the floor of patient contact. A defibrillator was applied by bystanders in $19(0.3 \%)$ of the cardiac arrests occurring on lower floors and $7(0.4 \%)$ of those occurring on the third floor or above $(p=0.7)$.

\section{Interpretation}

In this study, survival to hospital discharge after out-of-hospital cardiac arrest in private residences was better when the patient was located on a lower floor. Survival was negligible $(0.9 \%)$ for those living on floor 16 or above, and there were no survivors above floor 25 .

Our results are consistent with previous literature showing that 911 response time, measured from the time of the 911 call to arrival of the response vehicle on scene, is a significant predictor of patient survival after out-of-hospital cardiac arrest. ${ }^{9,10,19}$ However, those studies did not account for the time required for 911-initiated first responders to reach the patient's side and initiate care. Few previous studies have attempted to measure this extended interval..$^{11,13,14,20}$ Campbell and colleagues ${ }^{11}$ showed that prehospital response time was significantly longer when the time from arrival of 911initiated first responders on scene to patient contact was included. Morrison and colleagues ${ }^{14}$ observed a response delay of 1.5 minutes for all 911 emergency medical calls that occurred at or above the third floor in high-rise buildings in Toronto. Previous cardiac arrest research has found similar delays to patient care after arrival of 911-initiated first responders on scene. Campbell and colleagues ${ }^{20}$ found an average delay to defibrillation of 3.6 minutes from arrival of 911-initiated first responders on scene. However, they did not report on patient outcomes in relation to this delay. Our determination of the negative effect of a delay in reaching higher floors on patient outcomes after out-of-hospital cardiac arrest is novel and important.

Longer time from the arrival of 911-initiated first responders on scene to patient contact is one potential explanation for lower survival on the higher floors. This delay to patient contact could explain the smaller proportion of patients with 
an initial shockable rhythm found on the higher floors. After a patient collapses in cardiac arrest, deterioration from a shockable to a nonshockable rhythm occurs rapidly, ${ }^{21}$ and the presence of an initial shockable rhythm is one of the most important determinants of survival. ${ }^{22}$ With a rapidly deteriorating heart rhythm, and in the absence of defibrillation, cardiac arrests occurring on higher floors had a lower probability of survival because of the delay to patient contact by 911-initiated first responders. Another possible explanation for lower survival on higher floors is the potential for extended extrication times from the patient's residence. Lateef and colleagues $^{13}$ showed that higher vertical heights were associated with extended delays in extrica- tion time. Once a decision has been made to transport a patient who is in refractory cardiac arrest, there are prolonged periods during the extrication to the ambulance and en route to the hospital when the quality of CPR is suboptimal. ${ }^{23}$ As the patient is carried down stairs or is transported in an elevator, there is a shift in focus from providing continuous, high-quality CPR to removing the patient from the scene and getting him or her to the hospital quickly and safely. These disruptions in care could have a detrimental effect on patient outcome.

Although we observed a significant decrease in survival after cardiac arrest on higher floors, the same relation did not hold for the outcome of return of spontaneous circulation. The lack of an

Table 2: Unadjusted and adjusted analyses of variables associated with patient survival to hospital discharge

\begin{tabular}{|c|c|c|c|c|}
\hline \multirow[b]{2}{*}{ Variable } & \multicolumn{2}{|c|}{ No. $(\%)$ of patients* $\dagger$} & \multicolumn{2}{|c|}{ OR $(95 \% \mathrm{Cl})$} \\
\hline & $\begin{array}{c}\text { Survived } \\
n=300\end{array}$ & $\begin{array}{l}\text { Did not survive } \\
\qquad n=7538\end{array}$ & Unadjusted & Adjusted $¥$ \\
\hline Age, yr, mean $\pm S D$ & $60.1 \pm 16.3$ & $71.1 \pm 15.7$ & $0.96(0.958-0.97)$ & $0.96(0.95-0.97)$ \\
\hline Sex, male & $201 / 300(67.0)$ & $4647 / 7538(61.6)$ & $0.77(0.61-0.98)$ & $0.72(0.54-0.95)$ \\
\hline Event witnessed by bystander & $224 / 294(76.2)$ & $3007 / 7488(40.2)$ & $4.76(3.68-6.25)$ & $2.93(2.16-3.98)$ \\
\hline Bystander performed CPR & $136 / 300(45.3)$ & $2637 / 7538(35.0)$ & $1.55(1.23-1.94)$ & $1.07 \quad(0.83-1.39)$ \\
\hline 911 response time, $\min$, mean $\pm S D$ & $5.8 \pm 2.1$ & $6.2 \pm 2.3$ & $0.89(0.83-0.95)$ & $0.86 \quad(0.79-0.92)$ \\
\hline Initial shockable rhythm present & $219 / 297(73.7)$ & $1160 / 7502(15.5)$ & $14.65(11.39-19.02)$ & $10.68(7.98-14.29)$ \\
\hline $\begin{array}{l}\text { Time from arrival of } 911 \text { responder to } \\
\text { patient contact, min, mean } \pm \text { SD }\end{array}$ & $3.2 \pm 1.9$ & $3.5 \pm 2.2$ & $0.93(0.88-0.98)$ & NA \\
\hline
\end{tabular}

Table 3: Unadjusted and adjusted analyses of variables associated with return of spontaneous circulation (ROSC)

\begin{tabular}{|c|c|c|c|c|}
\hline Variable & $\begin{array}{c}\operatorname{ROSC} \\
n=2216\end{array}$ & $\begin{array}{l}\text { No ROSC } \\
n=5626\end{array}$ & Unadjusted & Adjusted $\dagger$ \\
\hline Age, yr, mean \pm SD & $71.0 \pm 15.4$ & $70.6 \pm 16.0$ & $1.00(0.99-1.00)$ & $1.00(1.00-1.01)$ \\
\hline Sex, male & $1325 / 2216(59.8)$ & $3525 / 5626(62.7)$ & $0.89(0.80-0.98)$ & $0.74(0.67-0.83)$ \\
\hline Bystander performed CPR & $889 / 2216(40.1)$ & $1884 / 5626$ (33.5) & $1.33(1.20-1.47)$ & $1.16(1.04-1.29)$ \\
\hline 911 response time, min, mean \pm SD & $6.1 \pm 2.3$ & $6.2 \pm 2.3$ & $0.99(0.97-1.01)$ & $0.98(0.95-1.00)$ \\
\hline Initial shockable rhythm present & $668 / 2212(30.2)$ & $711 / 5591 \quad(12.7)$ & $2.97(2.63-3.35)$ & $2.39(2.10-2.72)$ \\
\hline $\begin{array}{l}\text { Time from arrival of } 911 \text { responder to } \\
\text { patient contact, min, mean } \pm \text { SD }\end{array}$ & $3.3 \pm 2.3$ & $3.5 \pm 2.3$ & $0.97(0.95-0.99)$ & NA \\
\hline
\end{tabular}


association between vertical height and return of spontaneous circulation suggests that the mechanism for decreased survival is not failure of Advanced Cardiac Life Support treatment, but rather the damage related to prolonged response times to higher floors, even when circulation is ultimately restored.

Researchers investigating out-of-hospital cardiac arrests typically adjust for 911 response intervals from the time of emergency activation to arrival on scene, but they do not routinely account for the interval from arrival on scene to patient contact. Since the latter interval can lead to considerable delays in the initiation of patient care, it is important that future research include adjustments for this factor. As more high-rise buildings are built, in response to the demand for affordable condominiums and rental properties, the negative impact on community survival may increase. The 911 response time, from emergency activation to arrival of first responders on scene, will remain relatively constant, so long as traffic patterns do not change; however, the time from arrival on scene to initial patient contact may increase as more of the population comes to live at or above the third floor. Thus, 911 response time may diminish in importance as a determinant of survival, whereas the time to patient contact may become more important in predicting who lives and who dies after out-of-hospital cardiac arrest.

Identification and correction of barriers to 911-initiated first responders may help to improve the time to patient contact. One potential barrier is elevator access. Fire departments have a universal elevator access key, which gives firefighters sole access to elevators without public interference. In contrast, only rarely in prehospital care systems do paramedics have access to a universal elevator key. Availability of a universal key seems like a simple intervention, but it has remained unaddressed for decades. Perhaps quantifying the extent to which this barrier prolongs the interval to patient contact and affects outcomes will persuade municipal administrators to address this barrier to care. Conveying emergency alerts to building staff (e.g., security personnel) before the arrival of 911-initiated first responders may also reduce delays. Including local building staff in the emergency response process could minimize unnecessary delays in gaining access to the building and ensure that elevators are waiting on the ground floor at the arrival times of both fire department personnel and paramedics. Finally, optimization of placement of automated external defibrillators to maximize their availability for bystander use may improve outcomes after cardiac arrest in high-rise buildings. The rate of bystander use of automated external defibrillators was very low in this study. Because the presence of a shockable rhythm deteriorates over time, providing bystanders in high-rise buildings with easy access to automated external defibrillators may increase the use of these devices. Improving the accessibility of defibrillators could be accomplished by placing the devices on specific floors, in building lobbies or inside elevators, so that they can be easily delivered to the floor of the cardiac arrest, saving precious minutes and ensuring rapid defibrillation.

\section{Limitations}

Given the nature of retrospective observational studies, we could not determine a causal relation between survival and vertical height. The emergency medical services in this study were involved in the Resuscitation Outcomes Consortium network and as such were well established, with rigorous quality assurance and feedback mechanisms in place. Therefore, the results of this study may not be generalizable to other emergency medical services that lack similar oversight and monitoring of resuscitation performance. Because of limited information available from paramedic documentation, the third floor was used as the cutoff for examining the relation between vertical height and survival, but this may not represent the optimal cut-point for analysis. Very few cardiac arrests occurred on higher floors, and there were no survivors above floor 25 . This skewing of the data may have biased our analysis using floor of contact as a continuous variable. Finally, patient outcome data were missing for about $5 \%$ of the cases, although this is well within acceptable standards.

\section{Conclusion}

With continuing construction of high-rise buildings, it is important to understand the potential effect of vertical height on patient outcomes after out-of-hospital cardiac arrest. In our study, cardiac arrests that occurred on higher floors were associated with lower survival, and there were no survivors above floor 25 . These results may have been due to longer intervals from arrival of 911-initiated first responders to patient contact and lower rates of an initial shockable rhythm for patients on the higher floors. Interventions aimed at improving access to patients in highrise buildings may increase rates of survival.

\section{References}

1. Go AS, Mozaffarian D, Roger VL, et al. Heart disease and stroke statistics - 2013 update: a report from the American Heart Association. Circulation 2013;127:e6-245.

2. Nichol G, Thomas E, Callaway CW, et al. Regional variation in out-of-hospital cardiac arrest incidence and outcome. JAMA 2008;300:1423-31.

3. Larsen MP, Eisenberg MS, Cummins RO, et al. Predicting sur- 
vival from out-of-hospital cardiac arrest: a graphic model. Ann Emerg Med 1993;22:1652-8.

4. Valenzuela TD, Roe DJ, Cretin S, et al. Estimating effectiveness of cardiac arrest interventions: a logistic regression survival model. Circulation 1997;96:3308-13.

5. Drennan IR, Lin S, Thorpe KE, et al. The effect of time to defibrillation and targeted temperature management on functional survival after out-of-hospital cardiac arrest. Resuscitation 2014:85:1623-8.

6. Toronto, C, Ontario (code 3520005) [table]. In: National Household Survey (NHS) profile [2011 National Household Survey]. Ottawa: Statistics Canada; 2013. Cat no. 99-004-XWE. Available: www12.statcan.gc.ca/nhs-enm/2011/dp-pd/prof/index.cfm?Lang=E (accessed 2016 Jan. 11).

7. Backgrounder: 2011 census: marital status, families, households and dwelling characteristics. Toronto: City of Toronto; 2012

8. Profile Toronto: housing occupancy trends 1996-2011. Toronto: City of Toronto, City Planning Division; 2015. Available: www1.toronto.ca/City\%20Of\%20Toronto/City\%20Planning/SIPA /Files/pdf/H/HousingOccupancyTrends2011_Oct2015\%20FINAL _accessible.pdf (accessed 2015 Nov. 20).

9. O'Keeffe C, Nicholl J, Turner J, et al. Role of ambulance response times in the survival of patients with out-of-hospital cardiac arrest. Emerg Med J 2011;28:703-6.

10. Vukmir RB. Survival from prehospital cardiac arrest is critically dependent upon response time. Resuscitation 2006;69:229-34.

11. Campbell JP, Gratton MC, Salomone JA 3rd, et al. Ambulance arrival to patient contact: the hidden component of prehospital response time intervals. Ann Emerg Med 1993;22:1254-7.

12. Silverman RA, Galea S, Blaney S, et al. The "vertical response time": barriers to ambulance response in an urban area. Acad Emerg Med 2007;14:772-8.

13. Lateef F, Anantharaman V. Delays in the EMS response to and the evacuation of patients in high-rise buildings in Singapore. Prehosp Emerg Care 2000;4:327-32.

14. Morrison LJ, Angelini MP, Vermeulen MJ, et al. Measuring the EMS patient access time interval and the impact of responding to high-rise buildings. Prehosp Emerg Care 2005;9:14-8.

15. Jacobs I, Nadkarni V, Bahr J, et al. Cardiac arrest and cardiopulmonary resuscitation outcome reports: update and simplification of the Utstein templates for resuscitation registries. A statement for healthcare professionals from a task force of the International Liaison Committee on Resuscitation (American Heart Association, European Resuscitation Council, Australian Resuscitation Council, New Zealand Resuscitation Council, Heart and Stroke Foundation of Canada, InterAmerican Heart Foundation, Resuscitation Council of Southern Africa). Resuscitation 2004;63:233-49.

16. Morrison LJ, Nichol G, Rea TD, et al. Rationale, development and implementation of the Resuscitation Outcomes Consortium Epistry-Cardiac Arrest. Resuscitation 2008;78:161-9.

17. Lin S, Morrison LJ, Brooks SC. Development of a data dictionary for the Strategies for Post Arrest Resuscitation Care (SPARC) network for post cardiac arrest research. Resuscitation 2011;82:419-22.

18. Peel, RM, Ontario (code 3521) [table]. In: National Household Survey (NHS) profile [2011 National Household Survey]. Ottawa: Statistics Canada; 2013. Cat no. 99-004-XWE. Available: www12.statcan.gc.ca/nhs-enm/2011/dp-pd/prof/index. cfm?Lang=E (accessed 2016 Jan. 11)
19. Pell JP, Sirel JM, Marsden AK, et al. Effect of reducing ambulance response times on deaths from out of hospital cardiac arrest: cohort study. BMJ 2001;322:1385-8.

20. Campbell JP, Kroshus KS, Lindholm DJ, et al. Measuring the call-receipt-to-defibrillation interval: evaluation of prehospital methods. Ann Emerg Med 1995;26:697-701.

21. Renkiewicz GK, Hubble MW, Wesley DR, et al. Probability of a shockable presenting rhythm as a function of EMS response time. Prehosp Emerg Care 2014;18:224-30.

22. Sasson C, Rogers MA, Dahl J, et al. Predictors of survival from out-of-hospital cardiac arrest: a systematic review and meta-analysis. Circ Cardiovasc Qual Outcomes 2010;3:63-81.

23. Olasveengen TM, Wik L, Steen PA. Quality of cardiopulmonary resuscitation before and during transport in out-of-hospital cardiac arrest. Resuscitation 2008;76:185-90.

Affiliations: Rescu, Li Ka Shing Knowledge Institute (Drennan, Strum, Byers, Buick, Lin, Cheskes, Hu, Morrison), St. Michael's Hospital, Toronto, Ont.; Institute of Medical Science, Faculty of Medicine (Drennan), Department of Family and Community Medicine (Cheskes) and Department of Medicine (Morrison), University of Toronto, Toronto, Ont.; Sunnybrook Centre for Prehospital Medicine (Drennan, Buick, Cheskes), Sunnybrook Health Sciences Centre, Toronto, Ont.

Contributors: Ian Drennan, Ryan Strum, Adam Byers and Laurie Morrison conceived the study, and all of the authors contributed to its design. Ian Drennan, Adam Byers, Steve Lin and Samantha Hu acquired the data, and all authors contributed to analyzing and interpreting the data. Ian Drennan and Ryan Strum drafted the manuscript, and all authors revised the manuscript for important intellectual content, gave final approval of the version to be published and agreed to act as guarantors of the work.

Funding: This study was supported by grants from the Canadian Institutes of Health Research (CIHR), the Heart and Stroke Foundation of Canada and in-kind support from Rescu, Li Ka Shing Knowledge Institute, St. Michael's Hospital, Toronto. The RescuNet Epistry database was funded by the Laerdal Foundation, CIHR, the Heart and Stroke Foundation of Canada, Defence Research and Development Canada and the National Institutes of Health (US). Ian Drennan received personal support from the CIHR Banting and Best Doctoral Scholarship Award (GSD 140280). Laurie Morrison is the Robert and Dorothy Pitts Chair in Acute Care and Emergency Medicine, St. Michael's Hospital.

Acknowledgements: The authors thank Garrie Wright, deputy chief of Toronto Paramedic Services, for his contributions to the study's concept and design and his input to the interpretation of results. The authors also thank all 911initiated first responders working on the front line of patient care and their continued contributions to primary data collection in resuscitation research at Rescu. 